\title{
Brain delivery of small interfering ribonucleic acid and drugs through intranasal administration with nano-sized polymer micelles
}

This article was published in the following Dove Press journal:

Medical Devices: Evidence and Research

6 January 2015

Number of times this article has been viewed

\section{Takanori Kanazawa}

School of Pharmacy, Tokyo University of Pharmacy and Life Sciences,

Tokyo, Japan
Correspondence: Takanori Kanazawa School of Pharmacy, Tokyo University of Pharmacy and Life Sciences, I432-I Horinouchi, Hachioji,

Tokyo 192-0392, Japan

Tel/Fax+8I 426764493

Email kanazawa@toyaku.ac.jp
Abstract: Recently, the development of effective strategies for enhancing drug delivery to the brain has been a topic of great interest in both clinical and pharmaceutical fields. In this review, we summarize our studies evaluating nose-to-brain delivery of drugs and small interfering ribonucleic acids in combination with cell-penetrating peptide-modified polymer micelles. Our findings show that the use of polymer micelles with surface modification with Tat peptide in the intranasal administration enables the non-invasive delivery of therapeutic agents to the brain by increasing the transfer of the administered drug or small interfering ribonucleic acid to the central nervous system from the nasal cavity.

Keywords: nose-to-brain, polymer micelles, cell-penetrating peptide, intranasal administration, nucleic acid

\section{Introduction}

The development of novel therapeutic systems that would be effective against intractable central nervous system (CNS) diseases such as Alzheimer's disease, Parkinson's disease, and brain tumors is expected to bring about breakthroughs in the future. In order to achieve therapeutic effectiveness, novel drug delivery systems capable of reaching target tissues are needed, in addition to new drugs.

In general, the blood-brain barrier (BBB) poses a major challenge to the drug development efforts targeting CNS disorders, since it limits the distribution of systemically administered therapeutics to the CNS. ${ }^{1,2}$ Therefore, the development of effective delivery systems capable of achieving therapeutic drug concentrations in the brain is of major interest not only in pharmaceutical research but also in the clinical field. Recently developed methodologies for brain drug delivery have been characterized as either invasive or non-invasive. The invasive methodologies consist of direct drug delivery by means of intraventricular or intracerebral administration and temporary disruption of the BBB, allowing the drug to enter the CNS. ${ }^{3}$ Non-invasive methodologies involve systemic drug delivery that uses drug carriers to deliver agents through a receptor-mediated or adsorptive transcytosis, ${ }^{4}$ or alternatively, by bypassing the BBB by using the nose-to-brain route. ${ }^{5-9}$

Intranasal delivery is a recognized non-invasive method of direct delivery of drugs to the CNS. ${ }^{5-9}$ In general, drugs administered through the nose-to-brain route are widely distributed within the cerebral regions, typically by migrating to the olfactory nerve, which is located in the epithelial tissue of the nasal olfactory mucosa inside the nasal cavity. ${ }^{10-12}$ Migration also occurs along the trigeminal nerve in the nasal cavity respiratory mucosa. ${ }^{10-12}$ Transport may also occur through the capillaries, lymphatics, and 
cerebrospinal fluid present in the nasal mucosa or by nasal mucociliary movement. ${ }^{10-14}$ Low-molecular-weight drugs, ${ }^{15,16}$ peptides, ${ }^{10,17}$ proteins, ${ }^{18,19}$ small interfering ribonucleic acid (siRNA), ${ }^{20,21}$ and cells ${ }^{22-24}$ are reported to reach the CNS following intranasal delivery in animals. ${ }^{13}$ In addition, insulin, ${ }^{25-29}$ melanocortin, ${ }^{30-33}$ angiotensin II, ${ }^{34}$ vasopressin,,${ }^{35}$ and oxytocin $^{36-41}$ were shown to be directly delivered to the CNS following intranasal administration in humans. ${ }^{13,42}$ Intranasal administration also provides a painless and convenient route that could be used for self-administration of drugs by patients. $^{9-12,43}$ Therefore, nose-to-brain delivery is a highly versatile route, which, in combination with novel drugs being developed for treating intractable CNS diseases, is a promising approach for the treatment of disorders such as Alzheimer's disease, Parkinson's disease, and brain tumors. Furthermore, nano-sized drug carriers may improve nose-to-brain drug delivery by their capability to increase the stability of the encapsulated drug against chemical and biological degradation. The modification of the surface of nanocarriers with the incorporation of cell-penetrating peptides (CPPs) could further improve the specific drug delivery into the target cells. In this review, we will introduce our studies evaluating anti-cancer drugs and/or siRNAs' delivery to the brain using a combination of the nose-to-brain route and CPP-modified polymer micelles. ${ }^{44-47}$

\section{Polymeric micelles for nose-to brain delivery}

A key property of the amphiphilic block copolymer is its capability to form nano-sized micelles by self-assembly in a particular solvent. ${ }^{48-50}$ The nano-sized polymer micelles have been utilized as core-shell-type colloidal carriers for delivery of drugs and genes. ${ }^{51-53}$ The use of these carriers could increase the availability of drugs to the CNS, since their small diameter would potentially allow the nanoparticles to be transported transcellularly along the neurons to the brain through various endocytic pathways of sustentacular or neuronal cells present in the nasal membrane. ${ }^{11}$

We selected methoxypolyethylene glycol (MPEG)polycaprolactone (PCL) (MPEG-PCL) co-polymers, which can be used to form nano-sized micelles, as nanocarriers and prepared coumarin-loaded MPEG-PCL micelles at a range of diameters. At first, we examined the brain- and bio-distribution of fluorescein model drugs (coumarin) in rats following intranasal administration of coumarin-loaded MPEG-PCL micelles, ranging in diameter from 100 to $600 \mathrm{~nm} .{ }^{44}$ No significant difference was observed between micelles with diameters ranging from 200 to $600 \mathrm{~nm}$.
However, coumarin concentration in the brain following administration of coumarin-loaded PEG-PCL micelles with a diameter of $100 \mathrm{~nm}$ was significantly higher than that observed with micelles of a larger diameter, indicating that nano-sized polymer micelles with a diameter of around $100 \mathrm{~nm}$ can facilitate nasal absorption and increase the delivery of drugs into the CNS. In previous reports, smaller diameters may allow nanoparticles to be transported to the brain through various endocytic pathways of sustentacular or neuronal cells in the olfactory membrane. . $11,12,43,54$ Therefore, these findings indicate that small nanoparticles are the most promising for use in intranasal brain delivery. The coumarin concentrations in non-targeted tissues, such as the liver, heart, kidney, and spleen, were found to be lower than those measured in the brain, indicating that intranasal administration of drugs may decrease the risk of side effects in non-CNS tissues.

We compared the efficiency of brain delivery following intravenous and intranasal routes of administration of MPEG-PCL nanomicelles. ${ }^{44}$ As shown in Figure 1A, the amount of coumarin measured in brain tissue after intranasal administration was significantly higher than that observed after intravenous administration, providing further evidence that the intranasal route is an effective method for braintargeted drug delivery. Additionally, the duration of coumarin presence in blood circulation after intranasal administration was significantly shorter than that observed after intravenous injection (Figure 1B). In particular, we were only able to detect very low levels of coumarin in the blood of rats following intranasal administration of coumarin-loaded MPEG-PCL micelles, indicating that drugs loaded within the micelles are delivered intranasally directly to the brain without significant leakage into systemic circulation. However, coumarin was found to enter the systemic blood circulation soon after intranasal administration of coumarin solution alone. Therefore, administration of drug solution is associated with the risk of side effects resulting from toxic effects on non-CNS tissues. These findings suggest that intranasal delivery of drugs into brain tissue using nano-sized micelles is more likely to be effective and less likely to produce side effects in non-CNS tissues.

\section{CPP-modified polymeric micelles for nose-to-brain delivery}

The CPPs, which are cationic or amphiphilic molecules derived from sources such as the human immunodeficiency virus Tat protein and Drosophila antennapedia homeoprotein, can enhance the intracellular delivery of molecules. ${ }^{55} \mathrm{~A}$ basic domain of Tat was previously reported to be the minimum 

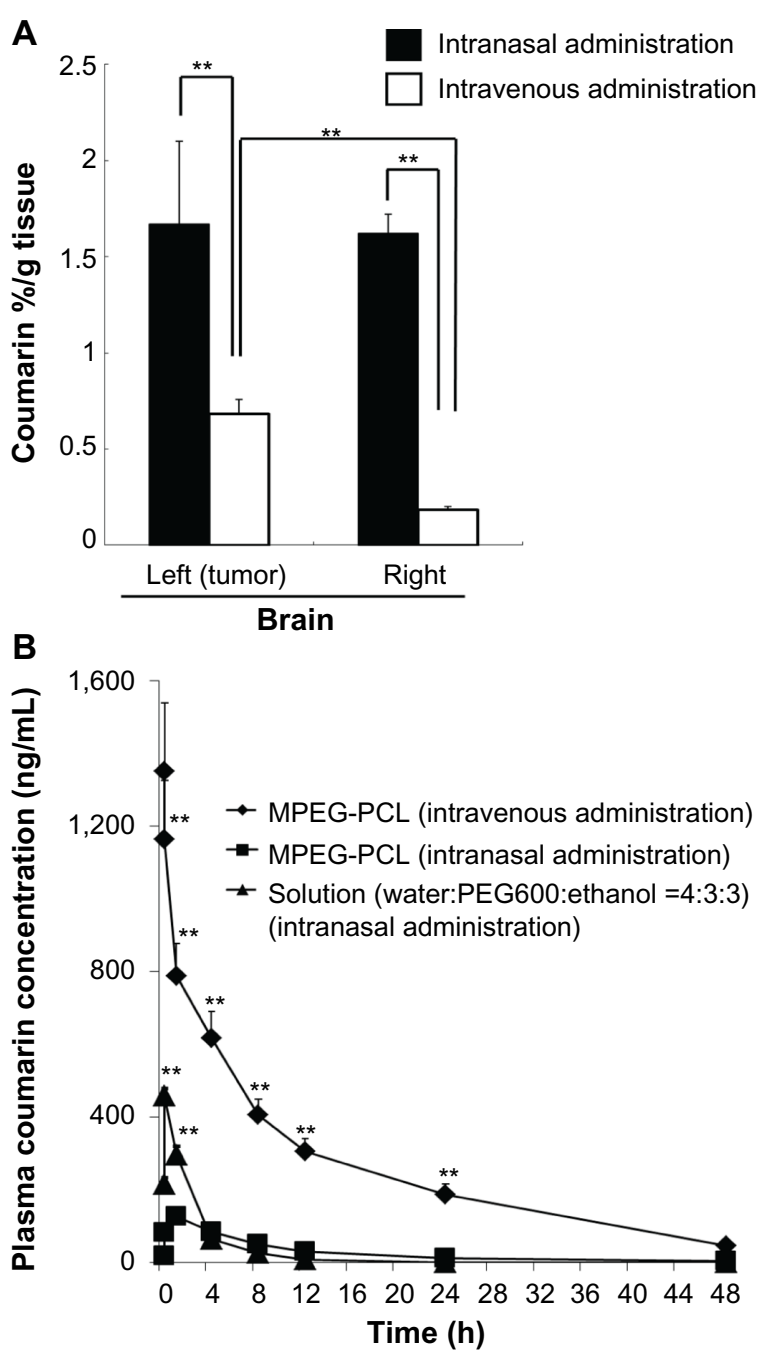

Figure I Distribution in brain tissue and blood concentration of coumarin after intravenous or intranasal administration of coumarin-loaded MPEG-PCL micelles. Notes: (A) Coumarin levels in the brain tissue of tumor-inoculated rats. Rats were sacrificed at I hour (following intranasal administration) or 24 hours (following intravenous administration) after the injection of coumarin-loaded MPEG-PCL micelles (dose corresponding to $20 \mu \mathrm{g}$ of coumarin). Each bar represents the mean \pm $\mathrm{SE}(\mathrm{n}=3)$. ${ }^{* *} \mathrm{P}<0.0 \mathrm{I}$. (B) Coumarin concentration in blood after administration of coumarin solution or coumarin-loaded MPEG-PCL (dose corresponding to $20 \mu \mathrm{g}$ of coumarin). Each point represents the mean $\pm S E(n=3)$. $* * P<0.0$ I versus intranasally administered MPEG-PCL.

Abbreviations: MPEG-PCL, methoxypolyethylene glycol-polycaprolactone; SE, standard error; h, hours; PEG, polyethylene glycol.

sequence responsible for cellular uptake mediated by the 11 -amino acid epitope YGRKKRRQRRR. ${ }^{56}$

Conjugation of the CPP to the surface on the nanocarriers was expected to increase the penetration across the nasal mucosa and target cell membrane into the brain. Therefore, we developed MPEG-PCL copolymers conjugated with Tat peptide (MPEG-PCL-Tat) and evaluated the potential for intranasal brain delivery of coumarin-loaded MPEG-PCL-Tat micelles (Figure 2). ${ }^{44}$

MPEG-PCL-Tat micelles showed high loading efficiency of coumarin. MPEG-PCL-Tat micelles had a smaller particle size (diameter of approximately $100 \mathrm{~nm}$ ) than the MPEG-PCL micelles without Tat and exhibited a positive charge, whereas MPEG-PCL micelles were negatively charged. These findings suggest that synthesized MPEG-PCL-Tat forms nanoparticles, possibly as polymer micelles. Tat is almost completely presented on the surface of the polymer micelles, since MPEG-PCL-Tat nanoparticles exhibited the positive charge of the Tat peptide.

We first compared the efficacy of nose-to-brain delivery between MPEG-PCL and MPEG-PCL-Tat micelles. At 4 hours following intranasal administration, coumarin concentration in brain tissue of rats administered coumarin-loaded MPEGPCL-Tat was significantly higher than in rats administered coumarin-loaded MPEG-PCL micelles. We subsequently determined the biodistribution of coumarin in rats after intranasal administration of coumarin-loaded MPEG-PCL-Tat micelles. As shown in Figure 3, the concentrations of coumarin in non-targeted tissues, such as liver, lung, heart, kidney, and spleen, in rats administered coumarin-loaded MPEG-PCL-Tat were lower than those measured following administration of coumarin solution without the polymer micelles. These findings indicate that CPP-modified nano-sized micelles can improve the nose-to-brain drug delivery.

\section{Nose-to-brain delivery of drug-loaded polymer micelles for treatment of brain tumors}

Although chemotherapy is widely applied in treatment of brain tumors, the outcomes continue to be unsatisfactory. In general, an increase in local anti-cancer drug concentration in the tumor tissue improves the outcome of the drug treatment. Therefore, we expected that the delivery of anticancer drugs using a combination of nose-to-brain route and MPEG-PCL-Tat micelles could increase the concentration of drugs at the intracranial tumors and suppress their growth. ${ }^{45}$ Therefore, we prepared MPEG-PCL and MPEG-PCL-Tat micelles loaded with the anti-cancer drug camptothecin (CPT). The particle size of CPT-loaded MPEG-PCL and MPEG-PCL-Tat micelles was slightly larger than that before CPT loading. CPT encapsulation efficiencies of MPEG-PCL and MPEG-PCL-Tat were determined to be around $60 \%$.

We evaluated the in vitro cytotoxicity in C6 rat glioma cells at a range of CPT concentrations. CPT-loaded Tat-modified MPEG-PCL exhibited stronger cytotoxicity than CPT-loaded MPEG-PCL. This observation may be due to the improved cellular uptake efficiency of CPT-loaded MPEG-PCL-Tat micelles by modification with CPP Tat on the surface of micelles. CPT-free MPEG-PCL-Tat did not exhibit 


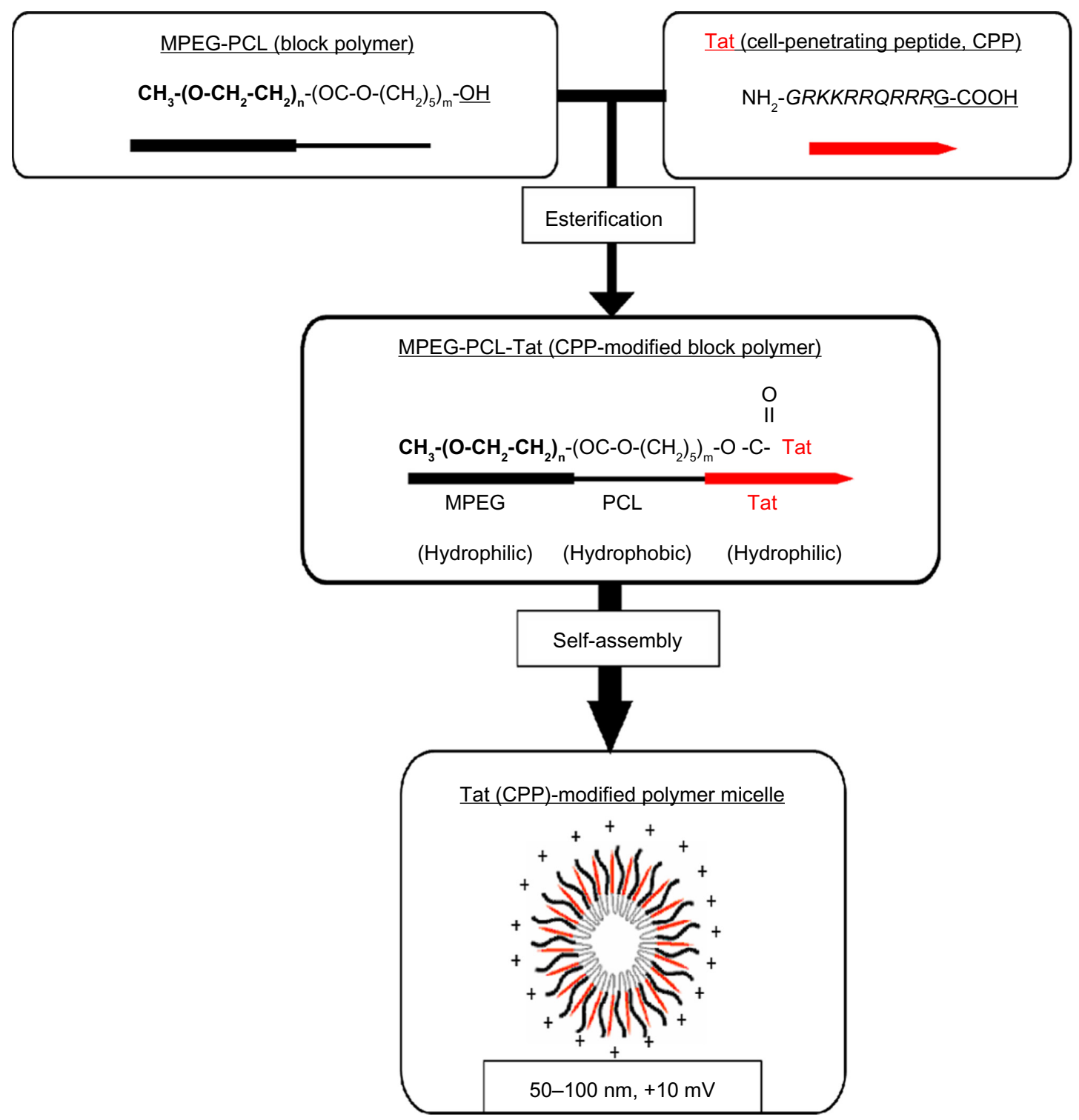

Figure 2 Structure of Tat-modified MPEG-PCL micelle.

Note: Carboxylic group in cell-penetrating peptide Tat was conjugated to the hydroxyl group in MPEG-PCL by esterification.

Abbreviations: MPEG, methoxypolyethylene glycol; PCL, polycaprolactone; CPP, cell-penetrating peptide; G, glycine; R, arginine; K, lysine; Q, glutamine.

any cytotoxicity at the higher concentrations tested. These results indicate that Tat-modified micelles strongly interact with the C6 rat glioma cells. Tat-modified polymer micelles would therefore be expected to result in greater delivery of CPT to tumor cells than polymer micelles without Tat.

We next determined the in vivo therapeutic effects in a rat model of intractable malignant glioma. CPT-loaded MPEG-PCL-Tat micelles exhibited high therapeutic efficiency after 7 days of continuous administration, unlike CPT-loaded MPEG-PCL. This observation suggests that Tat-modified MPEG-PCL micelles may be more effective because of high penetration of small molecule anti-cancer drugs across the nasal epithelium. The specificity achieved with intranasal delivery appears to be superior to the results obtained with the administration of simple CPT solution, which reportedly results in high drug levels in the systemic circulation.

\section{siRNA delivery to the brain using nose-to-brain administration and CPP-modified polymer micelles}

siRNA has great potential as a therapeutic agent against CNS diseases. ${ }^{57-61}$ Although the potential of using siRNA-based therapies against $\mathrm{CNS}$ disorders has been successfully demonstrated through in vitro studies, the function of BBB poses a major challenge to the drug development efforts aimed at treating CNS disorders. Therefore, the development of effective strategies that would enhance siRNA delivery to the brain is of great interest for both the clinical and pharmaceutical fields. In order to improve 


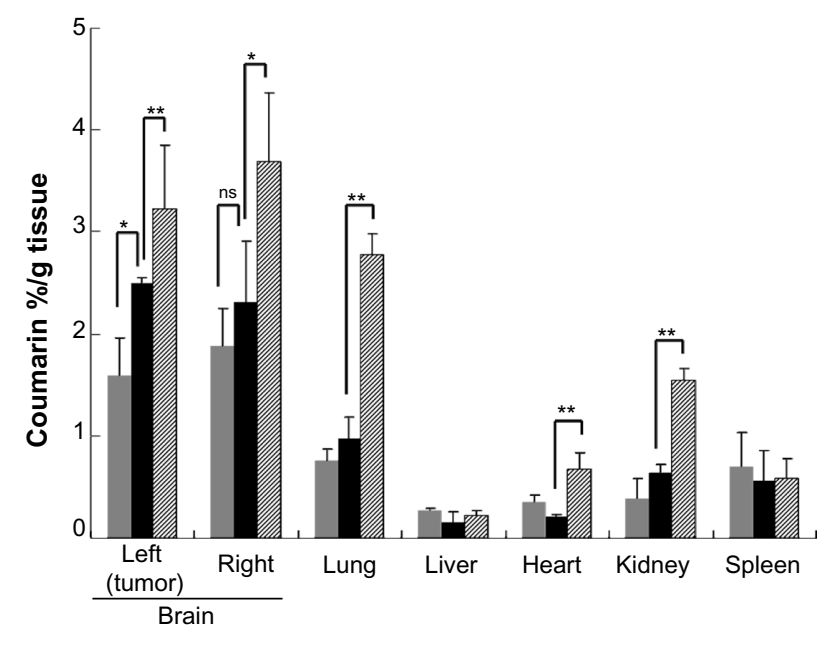

Figure 3 Biodistribution of coumarin in rats after intranasal administration of coumarin-loaded MPEG-PCL-Tat micelles.

Notes: Rats were sacrificed I hour $(\square)$ or 4 hours $(\square)$ after injection of coumarin-loaded MPEG-PCL-Tat micelles, or I hour (שיד) following the injection of coumarin solution (dose corresponding to $20 \mu \mathrm{g}$ of coumarin). Each bar represents the mean $\pm S E(n=3)$. ${ }^{n s} P>0.05, * * P<0.01, * P<0.05$.

Abbreviations: MPEG-PCL, methoxypolyethylene glycol-polycaprolactone; SE, standard error; ns, not significant.

the efficiency of brain siRNA delivery systems, we developed an approach that combines siRNA nose-to-brain delivery system with CPP-modified nano-sized micelles. ${ }^{46}$

Significantly higher brain penetration was observed following intranasal administration of MPEG-PCL-Tat to rats than with intravenous injection, suggesting that superior delivery of nucleic acid to the brain is possible using peptide-modified micelles. Additionally, in our study of the mechanisms that promote nucleic acid transfer to the brain following intranasal administration using MPEG-PCL-Tat, we focused on the olfactory and trigeminal nerves, which are the two reported pathways of drug transport to the brain from the nasal cavity. First, nasal mucosal tissue was observed 15 minutes after intranasal administration of naked siRNA and complexes of siRNA with MPEG-PCL-Tat micelles. As shown in Figure 4, animals administered siRNA complexed with MPEG-PCL-Tat exhibited more intense fluorescence in the mucosal epithelial surface and lamina propria mucosae than the animals administered naked siRNA, indicating that MPEG-PCL-Tat exhibits high mucosal penetration. Next, we assessed the distribution of the siRNA to the olfactory bulb after intranasal administration and found a significantly higher transfer of the siRNA to the olfactory bulb in the MPEG-PCLTat-treated animals, as compared to those administered the naked siRNA (Figure 5). This finding suggests that the use of MPEG-PCL-Tat may increase the transfer of nucleic acid to the brain via the olfactory nerve pathway in the nasal mucosal tissue. Additionally, the observation of the trigeminal nerve after intranasal administration detected higher transfer of the

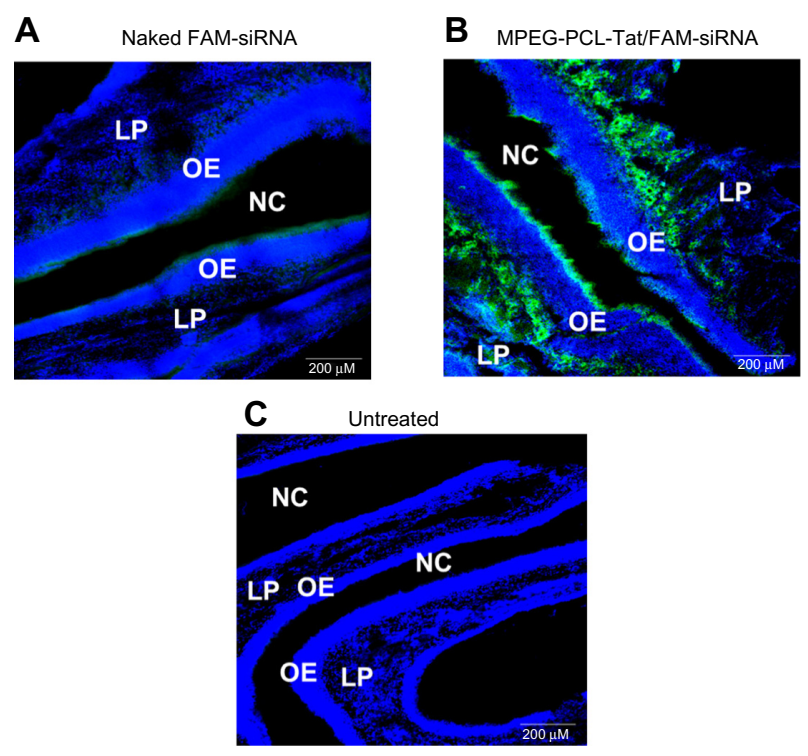

Figure 4 Distribution of FAM-siRNA in slices of nasal mucosa after intranasal administration of naked FAM-siRNA and MPEG-PCL-Tat/FAM-siRNA.

Notes: Rats were sacrificed 15 minutes after intranasal administration of naked FAMsiRNA (A) or MPEG-PCL-Tat/FAM-siRNA (B) (dose corresponding to $40 \mu \mathrm{g}$ of FAMsiRNA, N/P=15). Untreated rats $(\mathbf{C})$ were sacrificed at the same time point. Olfactory mucosa tissue was enucleated. Blue fluorescence represents the nuclei stained by Hoechst, and green fluorescence represents FAM-siRNA. Scale bar $=200 \mu \mathrm{m}$. 6-Carboxyfluorescein-aminohexyl (FAM)-siRNA (Cosmo Bio Co., Ltd., Tokyo, Japan) as a fluorescent-labeled siRNA.

Abbreviations: OE, olfactory mucosa epithelium; LP, lamina propria; NC, nasal cavity; MPEG-PCL, methoxypolyethylene glycol-polycaprolactone; N/P, ratio of amine to nucleic acid; FAM, 6-Carboxyfluorescein-aminohexyl; siRNA, small interfering ribonucleic acid.

fluorescein-labeled model siRNA (dextran, molecular weight: $10,000)$ to the trigeminal nerve in the MPEG-PCL-Tat group than in animals administered fluorescein-labeled model siRNA alone. This observation suggests that, as with the olfactory nerve, MPEG-PCL-Tat may also enable the nucleic acid to be delivered to the brain via the trigeminal nerve. Figure 6 demonstrates the distribution in the whole brain, from olfactory bulb to the brainstem, over time, measured following intravenous or intranasal administration of Anionic dextran labeled with Alexa Fluor ${ }^{\circledR} 678$ (molecular weight: 10,000), (Alexa-dextran; Life Technologies, Carlsbad, MA, USA) model siRNA with and without MPEG-PCL-Tat. While the fluorescence of Alexa-labeled model siRNA did not distribute in the whole brain following intravenous administration, high accumulation was observed in the olfactory bulb 15 minutes after administration via the nose-to-brain pathway, both with or without MPEG-PCL-Tat. One hour after intranasal administration with MPEG-PCL-Tat, high accumulation was observed in the rostral brain tissue that appeared to follow the distribution trend into the olfactory bulb, while the caudal brain tissue and brain stem exhibited a high concentration that appeared to follow a distribution trend into the trigeminal nerve. Taken together, these observations suggest that, once transported 


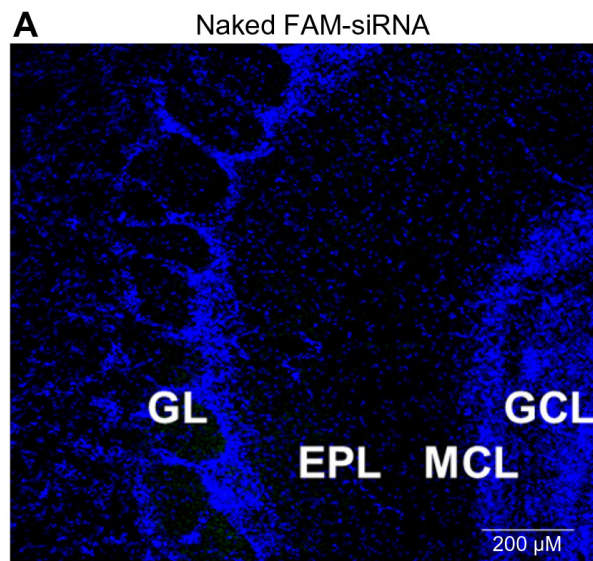

B MPEG-PCL-Tat/FAM-siRNA

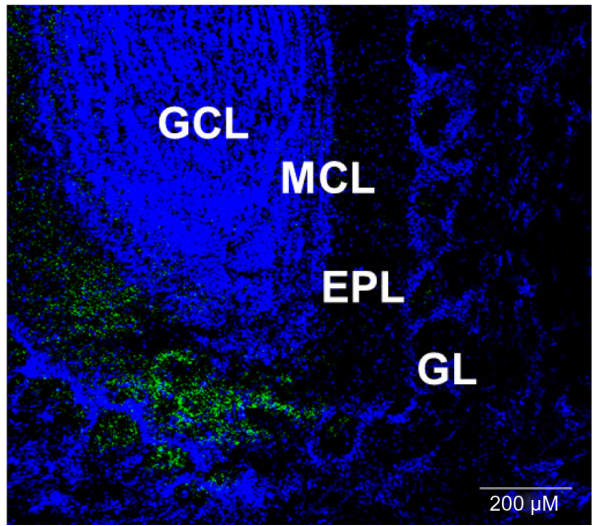

Figure 5 siRNA distribution in the olfactory bulb tissue after intranasal administration.

Notes: Rats were sacrificed 15 minutes after intranasal administration of naked FAM-siRNA (A) or MPEG-PCL-Tat/FAM-siRNA (B) (dose corresponding to $40 \mu \mathrm{g}$ of FAM-siRNA, N/P=15). Each olfactory bulb was enucleated. Blue fluorescence represents the nuclei stained by Hoechst, and green fluorescence represents FAMsiRNA. Scale bar $=200 \mu \mathrm{m}$. 6-Carboxyfluorescein-aminohexyl (FAM)-siRNA (Cosmo Bio Co., Ltd., Tokyo, Japan) as a fluorescent-labeled siRNA.

Abbreviations: GL, glomerular layer; $\mathrm{EPL}$, external plexiform layer; $\mathrm{MCL}$, mitral cell layer; GCL, granule cell layer; siRNA; small interfering ribonucleic acid; MPEG-PCL, methoxypolyethylene glycol-polycaprolactone; N/P, ratio of amine to nucleic acid; FAM, 6-Carboxyfluorescein-aminohexyl (FAM)-siRNA (Cosmo Bio Co., Ltd., Tokyo, Japan) as a fluorescent-labeled siRNA.

to the olfactory bulb and brain stem via the olfactory nerve and trigeminal nerve pathways, nucleic acid is subsequently transported to other brain tissues. This shows that the proposed system of delivery of nucleic acid to the brain by intranasal administration using MPEG-PCL-Tat relies primarily on an increase in the transfer of nucleic acid to the brain via the olfactory nerve and trigeminal nerve pathways.

\section{Co-delivery of siRNA and drugs using nose-to-brain and CPP-modified polymer micelles for treatment of brain tumors}

We investigated intranasal delivery of siRNA/drug coloaded MPEG-PCL-Tat micelles using a rat model of

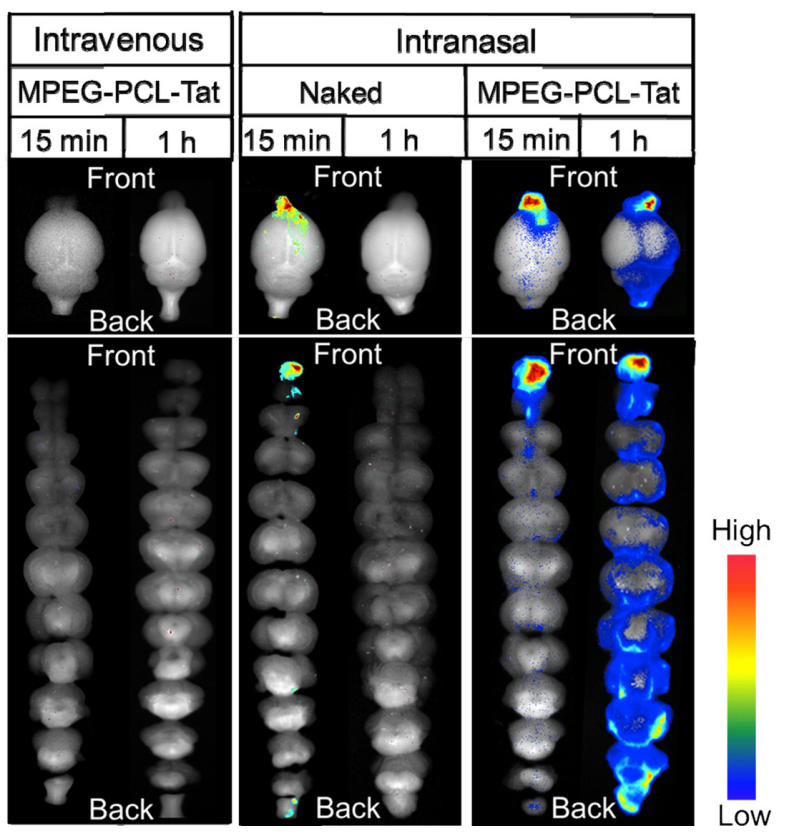

Figure 6 Dynamics of MPEG-PCL-Tat complex in brain tissue following intranasal or intravenous administration.

Notes: Rats were sacrificed and brains were enucleated at each point after intranasal or intravenous administration of naked Alexa-dextran or Alexa-dextran/ MPEG-PCL-Tat (dose corresponding to $40 \mu \mathrm{g}$ of Alexa-dextran). Each sample was observed using Maestro ${ }^{\mathrm{TM}}$ (Kurabo, Industries, Ltd., Osaka, Japan). Anionic dextran labeled with Alexa Fluor ${ }^{\circledast} 678$ (molecular weight: 10,000), (Alexa-dextran; Life Technologies, Carlsbad, MA, USA).

Abbreviations: MPEG-PCL, methoxypolyethylene glycol-polycaprolactone; $h$, hour; min, minutes.

malignant glioma. ${ }^{47}$ The MPEG-PCL-Tat/siRNA complex and CPT-loaded MPEG-PCL-Tat/siRNA complex exhibited micelle diameters of approximately $60-200 \mathrm{~nm}$, with a tendency of size to decrease as the ratio of amine to nucleic acid (N/P ratio) increased. The zeta-potential of these complexes was found to increase with increasing N/P ratio. Furthermore, on evaluation by the SYBR Green exclusion assay (Takara Bio Inc., Shiga, Japan), the fluorescence intensity from siRaf-1 of MPEG-PCL-Tat/siRaf-1 complexes strongly decreased at an N/P ratio of 1 (10\%), compared with that of naked siRNA (100\%). These results indicate that MPEGPCL-Tat and CPT-loaded MPEG-PCL-Tat could form a stable complex with siRNA.

We subsequently determined the cellular uptake of siRNA and evaluated its in vitro cytotoxicity in rat glioma $\mathrm{C} 6$ cells. The intracellular siRNA uptake of siRNA/MPEG-PCL-Tat complexes increased with increasing N/P ratio, with the highest intracellular uptake efficacy observed with the complexes at an N/P ratio of 30 . To evaluate the in vitro transfection efficiency of the MPEG-PCL-Tat/siRNA complexes at an N/P ratio of 30, we assessed the cytotoxicity induced by Raf-1 gene silencing in C6 cells using the WST- 8 assay (Dojindo Laboratories, Kumamoto, Japan). These results showed that 
the cell viability significantly decreased with an increase in the concentration of Raf-1 siRNA (siRaf-1), indicating that MPEG-PCL-Tat/siRaf-1 induces cell death in rat glioma cells due to the high cellular uptake of siRaf-1 by MPEGPCL-Tat carrier.

We finally evaluated the in vivo therapeutic effects in glioma model rats of intranasally administered naked siRaf-1 without any micelles, MPEG-PCL-Tat/control siRNA, MPEG-PCL-Tat/ siRaf-1 complex, CPT-loaded-MPEG-PCL-Tat micelles/ control siRNA complex, and CPT-loaded MPEG-PCL-Tat micelles/siRaf-1 complexes. MPEG-PCL-Tat/siRaf-1 complex, CPT-loaded-MPEG-PCL-Tat micelles/control siRNA complex, and CPT-loaded MPEG-PCL-Tat micelles/ siRaf-1 complexes showed high therapeutic efficacy after 7 days of continuous delivery. These results indicate that MPEG-PCL-Tat improved the delivery of siRNAs and CPT to the brain, and showed a marked prolongation of the mean survival period. No cytotoxicity was detected in neuronal cells and no signs of macroscopic damage in rat nasal mucosa, olfactory and trigeminal nerves, and brain tissue were observed.

\section{Conclusion and future perspectives}

Through the review, we have shown that the use of polymer micelles with surface-loaded Tat peptide in the intranasal administration of drugs and siRNAs enables the non-invasive delivery of therapeutic agents to the brain by increasing the transfer of drug or siRNA to the CNS from the nasal cavity. This improvement in delivery is not as pronounced with naked micelles. We feel that this review offers important insight that can be applied to improve drug delivery to the brain and shows the potential of using drug-loaded surfaceloaded polymer micelles against intractable neuropsychiatric disorders, brain tumors, and cerebral infarction.

\section{Acknowledgments}

This work was supported in part by Grant-in-Aid for Young Scientists (B) (25870767) from the Japan Society for the Promotion of Science (JSPS) and in part by a Grant for Private Universities provided by the Promotion and Mutual Aid Corporation for Private Schools of Japan.

\section{Disclosure}

The author declares no conflicts of interest in this work.

\section{References}

1. Pathan SA, Iqbal Z, Zaidi SM, et al. CNS drug delivery systems: novel approaches. Recent Pat Drug Deliv Formul. 2009;3(1):71-89.

2. Xia H, Gao X, Gu G, et al. Low molecular weight protamine-functionalized nanoparticles for drug delivery to the brain after intranasal administration. Biomaterials. 2011;32(36):9888-9898.
3. Tosi G, Costantino L, Rivasi F, et al. Targeting the central nervous system: in vivo experiments with peptide-derivatized nanoparticles loaded with loperamide and rhodamine-123. J Control Release. 2007; 122(1):1-9.

4. Kreuter J. Nanoparticulate system for brain delivery of drugs. Adv Drug Deliv Rev. 2001;47(1):65-81.

5. Dhuria SV, Hanson LR, Frey WH 2nd. Intranasal delivery to the central nervous system: mechanisms and experimental considerations. J Pharm Sci. 2010;99(4):1654-1673.

6. Thorne RG, Frey WH 2nd. Delivery of neurotrophic factors to the central nervous system: pharmacokinetic considerations. Clin Pharmacokinet. 2001;40(12):907-946.

7. Illum L. Transport of drugs from the nasal cavity to the central nervous system. Eur J Pharm Sci. 2000;11(1):1-18.

8. Illum L. Is nose-to-brain transport of drugs in man a reality? J Pharm Pharmacol. 2004;56(1):3-17.

9. Mistry A, Stolnik S, Illum L. Nanoparticles for direct nose-to-brain delivery of drugs. Int J Pharm. 2009;379(1):146-157.

10. Thorne RG, Pronk GJ, Padmanabhan V, Frey WH 2nd. Delivery of insulin-like growth factor-1 to the rat brain and spinal cord along olfactory and trigeminal pathways following intranasal administration. Neuroscience. 2004;127(2):481-496.

11. Lochhead JJ, Thorne RG. Intranasal delivery of biologics to the central nervous system. Adv Drug Deliv Rev. 2011;64(7):614-628.

12. Djupesland PG, Messina JC, Mahmoud RA. The nasal approach to delivering treatment for brain diseases: an anatomic, physiologic, and delivery technology overview. Ther Deliv. 2014;5(6):709-733.

13. Chapman CD, Frey WH 2nd, Craft S, et al. Intranasal treatment of central nervous system dysfunction in humans. Pharm Res. 2012;30(10): 2475-2484.

14. Merkus FW, Van Den Berg MP. Can nasal drug delivery bypass the blood-brain barrier?: questioning the direct transport theory. Drugs $R D$. 2007;8(3):133-144.

15. Shingaki T, Inoue D, Furubayashi T, et al. Transnasal delivery of methotrexate to brain tumors in rats: a new strategy for brain tumor chemotherapy. Mol Pharm. 2010;7(5):1561-1568.

16. Sakane T, Yamashita S, Yata N, Sezaki H. Transnasal delivery of 5-fluorouracil to the brain in the rat. J Drug Target. 1999;7(3):233-240.

17. Thorne RG, Hanson LR, Ross TM, Tung D, Frey WH 2nd. Delivery of interferon-beta to the monkey nervous system following intranasal administration. Neuroscience. 2008;15(3)2:785-797.

18. Alcalá-Barraza SR, Lee MS, Hanson LR, McDonald AA, Frey WH 2nd, McLoon LK. Intranasal delivery of neurotrophic factors BDNF, CNTF, EPO, and NT-4 to the CNS. J Drug Target. 2010; 18(3):179-190.

19. Yang JP, Liu HJ, Cheng SM, et al. Direct transport of VEGF from the nasal cavity to brain. Neurosci Lett. 2009;449(2):108-111.

20. Renner DB, Frey WH 2nd, Hanson LR. Intranasal delivery of siRNA to the olfactory bulbs of mice via the olfactory nerve pathway. Neurosci Lett. 2012;513(2):193-197.

21. Nishina K, Mizusawa H, Yokota T. Short interfering RNA and the central nervous system: development of nonviral delivery systems. Expert Opin Drug Deliv. 2013;10(3):289-292.

22. Danielyan L, Schäfer R, von Ameln-Mayerhofer A, et al. Intranasal delivery of cells to the brain. Eur J Cell Biol. 2009;88(6):315-324.

23. Danielyan L, Schäfer R, von Ameln-Mayerhofer A, et al. Therapeutic efficacy of intranasally delivered mesenchymal stem cells in a rat model of Parkinson disease. Rejuvenation Res. 2011;14(1):3-16.

24. van Velthoven CT, Sheldon RA, Kavelaars A, et al. Mesenchymal stem cell transplantation attenuates brain injury after neonatal stroke. Stroke. 2013;44(5):1426-1432.

25. Kern W, Born J, Schreiber H, Fehm HL. Central nervous system effects of intranasally administered insulin during euglycemia in men. Diabetes. 1999;48(3):557-563.

26. Benedict $\mathrm{C}$, Hallschmid $\mathrm{M}$, Hatke A, et al. Intranasal insulin improves memory in humans. Psychoneuroendocrinology. 2004;29(10): 1326-1334. 
27. Benedict C, Kern W, Schultes B, Born J, Hallschmid M. Differential sensitivity of men and women to anorexigenic and memory-improving effects of intranasal insulin. J Clin Endocrinol Metab. 2008;93(4): 1339-1344.

28. Benedict C, Brede S, Schioth HB, et al. Intranasal insulin enhances postprandial thermogenesis and lowers postprandial serum insulin levels in healthy men. Diabetes. 2011;60(1):114-118.

29. Reger MA, Watson GS, Green PS, et al. Intranasal insulin improves cognition and modulates beta-amyloid in early AD. Neurology. 2008;70(6):440-448.

30. Born J, Lange T, Kern W, McGregor GP, Bickel U, Fehm HL. Sniffing neuropeptides:a transnasal approach to the human brain. Nat Neurosci. 2002;5(6):514-516.

31. Fehm HL, Smolnik R, Kern W, McGregor GP, Bickel U, Born J. The melanocortin melanocyte-stimulating hormone/adrenocorticotropin(4-10) decreases body fat in humans. J Clin Endocrinol Metabol. 2001;86(3): 1144-1148.

32. Smolnik R, Perras B, Molle M, Fehm HL, Born J. Event-related brain potentials and working memory function in healthy humans after singledose and prolonged intranasal administration of adrenocorticotropin 4-10 and desacetyl-alpha-melanocyte stimulating hormone. J Clin Psychopharmacol. 2000;20(4):445-454.

33. Hallschmid M, Smolnik R, McGregor G, Born J, Fehm HL. Overweight humans are resistant to the weight-reducing effects of melanocortin 4-10. J Clin Endocrinol Metabol. 2006;91(2):522-525.

34. Derad I, Willeke K, Pietrowsky R, Born J, Fehm HL. Intranasal angiotensin II directly influences central nervous regulation of blood pressure. Am J Hypertens. 1998;11(8 Pt 1):971-977.

35. Pietrowsky R, Struben C, Molle M, Fehm HL, Born J. Brain potential changes after intranasal vs. intravenous administration of vasopressin: evidence for a direct nose-brain pathway for peptide effects in humans. Biol Psychiatry. 1996;39(5):332-340.

36. Kosfeld M, Heinrichs M, Zak PJ, Fischbacher U, Fehr E. Oxytocin increases trust in humans. Nature. 2005;435(7042):673-676.

37. Kirsch P, Esslinger C, Chen Q, et al. Oxytocin modulates neural circuitry for social cognition and fear in humans. J Neurosci. 2005;25(49): 11489-11493.

38. Domes G, Heinrichs M, Michel A, Berger C, Herpertz SC. Oxytocin improves "mind-reading" in humans. Biol Psychiatry. 2007;61(6): 731-733.

39. Heinrichs M, Baumgartner T, Kirschbaum C, Ehlert U. Social support and oxytocin interact to suppress cortisol and subjective responses to psychosocial stress. Biol Psychiatry. 2003;54(12):1389-1398.

40. Labuschagne I, Phan KL, Wood A, et al. Oxytocin attenuates amygdala reactivity to fear in generalized social anxiety disorder. Neuropsychopharmacology. 2010;35(12):2403-2413.

41. Guastella AJ, Einfeld SL, Gray KM, et al. Intranasal oxytocin improves emotion recognition for youth with autism spectrum disorders. Biol Psychiatry. 2010;67(7):692-694.

42. Lalatsa A, Schatzlein AG, Uchegbu IF. Strategies to deliver peptide drugs to the brain. Mol Pharm. 2014;11(4):1081-1093.

43. Dhanda DS, Frey WH 2nd, Leopold D, Kompella UB. Approaches for drug deposition in the human olfactory epithelium. Drug Deliv Technol. 2005;5:64-72.

44. Kanazawa T, Taki H, Tanaka K, Takashima Y, Okada H. Cell-penetrating peptide-modified block copolymer micelles promote direct brain delivery via intranasal administration. Pharm Res. 2011;28(9): 2130-2139.

Medical Devices: Evidence and Research

\section{Publish your work in this journal}

Medical Devices: Evidence and Research is an international, peerreviewed, open access journal that focuses on the evidence, technology, research, and expert opinion supporting the use and application of medical devices in the diagnosis, treatment and management of clinical conditions and physiological processes. The identification of novel
45. Taki H, Kanazawa T, Akiyama F, Takashima Y, Okada H. Intranasal delivery of camptothecin-loaded Tat-modified nanomicells for treatment of intracranial brain tumors. Pharmaceuticals. 2012;5(10): 1092-1102.

46. Kanazawa T, Akiyama F, Kakizaki S, Takashima Y, Seta Y. Delivery of siRNA to the brain using a combination of nose-to-brain delivery and cell-penetrating peptide-modified nano-micelles. Biomaterials. 2013;34(36):9220-9226.

47. Kanazawa T, Morisaki K, Suzuki S, Takashima Y. Prolongation of life in rats with malignant glioma by intranasal siRNA/drug codelivery to the brain with cell-penetrating peptide-modified micelles. Mol Pharm. 2014;11(5):1471-1478.

48. Allen C, Yu Y, Maysinger D, Eisenberg A. Poly caprolactoneb-poly(ethylene oxide) block copolymer micelles as a novel drug delivery vehicle for neurotrophic agents FK506 and L-685,818. Bioconjug Chem. 2008;9(5):564-572.

49. Otsuka H, Nagasaki Y, Kataoka K. PEGylated nanoparticles for biological and pharmaceutical applications. Adv Drug Deliv Rev. 2003;55(3):403-419.

50. Jang JS, Kim SY, Lee SB, Kim KO, Han JS, Lee YM. Poly(ethylene glycol)/poly ( $\varepsilon$-caprolactone) diblock copolymeric nanoparticles for non-viral gene delivery: the role of charge group and molecular weight in particle formation, cytotoxicity and transfection. $J$ Control Release. 2006;113(2):173-182.

51. Shin IG, Kim SY, Lee YM, Cho CS, Sung YK. Methoxy poly(ethylene gricol)/E-caprolactone amphiphilic block copolymeric micelle containing indomethacin. I. Preparation and characterization. J Control Release. 1998;51(1):1-11.

52. Kakizawa Y, Kataoka K. Block copolymer micelles for delivery of gene and related compounds. Adv Drug Deliv Rev. 2002;54(2):203-222.

53. Sharma R, Lee JS, Bettencourt RC, Xiao C, Konieczny SF, Won YY. Effects of the incorporation of hydrophobic middle block into a PEG-polycation diblock copolymer on the physicochemical and cell interaction properties of the polymer-DNA complexes. Biomacromolecules. 2008;9(11):3294-3307.

54. Brooking J, Davis SS, Illum L. Transport of nanoparticles across the rat nasal mucosa. J Drug Target. 2001;9(4):267-279.

55. Yang S, Coles DJ, Esposito A, Mitchell DJ, Toth I, Minchin RF. Cellular uptake of self-assembled cationic peptide-DNA complex: multifunctional role of the enhancer chloroquine. J Control Release. 2009;135(2):159-165.

56. Rajagopalan R, Xavier J, Rangaraj N, Rao NM, Gopal V. Recombinant fusion proteins TAT-Mu. $\mathrm{Mu}$ and $\mathrm{Mu}-\mathrm{Mu}$ mediate efficient non viral gene delivery. J Gene Med. 2007;9(4):275-286.

57. Chen S, Ge X, Chen Y, Lv N, Liu, Yuan W. Advances with RNA interference in Alzheimer's disease research. Drug Des Devel Ther. 2013;7:117-125.

58. Piwecka M, Rolle K, Wyszko E, et al. Nucleic acid-based technologies in therapy of malignant gliomas. Curr Pharm Biotechnol. 2011;12(11):1805.

59. Orlacchio A, Bernardi G, Orlacchio A, Martino S. RNA interference as a tool for Alzheimer's disease therapy. Mini Rev Med Chem. 2007;7(11):1166-1176.

60. Pappas TC, Bader AG, Andruss BF, Brown D, Ford LP. Applying small RNA molecules to the directed treatment of human diseases: realizing the potential, Expert Opin Ther Targets. 2008;12(1):115-127.

61. Dykxhoorn DM. RNA interference as an anticancer therapy:patent perspective. Expert Opin Ther Pat. 2009;19(4):475-491.

\section{Dovepress}

devices and optimal use of existing devices which will lead to improved clinical outcomes and more effective patient management and safety is a key feature. The manuscript management system is completely online and includes a quick and fair peer-review system. Visit http://www. dovepress.com/testimonials.php to read real quotes from authors. 